\title{
Quantum electrodynamics in the gravitational field of a cosmic string
}

\author{
Vladimir D. Skarzhinsky ${ }^{1,3}$, Diego D. Harari ${ }^{2}$ and Ulf Jasper ${ }^{3}$ \\ ${ }^{1}$ P. N. Lebedev Physical Institute \\ Leninsky prospect 53, Moscow 117924, Russia \\ ${ }^{2}$ Departamento de Física, Facultad de Ciencias Exactas y Naturales \\ Universidad de Buenos Aires, Ciudad Universitaria - Pab.1 \\ 1428 Buenos Aires, Argentina \\ ${ }^{3}$ Fakultät für Physik der Universität Konstanz \\ Postfach 5560, D 78434 Konstanz, Germany
}

\begin{abstract}
We evaluate the cross section for electron-positron pair production by a single high energy photon in the space-time of a static, straight cosmic string. Energy and momentum conservation precludes this process in empty Minkowski space. It happens around a cosmic string, in spite of the local flatness of the metric, as a consequence of the conical structure of space. Previous results based on a simplified model with scalar fields are here extended to the realistic QED case. Analytic expressions are found in three different regimes: near the threshold, at energies much larger than the electron rest mass $M$, and at ultrahigh energies, much larger than $M / \delta$, with $\delta$ the string mass per unit length in Planck units.
\end{abstract}

To appear in Physical Review D 


\section{Introduction}

Electron-positron pair production by a single photon with energy higher than twice the electron rest mass does not occur in empty Minkowski space, as dictated by conservation of linear momentum. It is a rather common process, though, in the vicinity of atomic nuclei, their inhomogeneous Coulomb field providing the requisite momentum. The cross section for this process is known since the earlier times of QED. ${ }^{[1]}$ The aim of this paper is to evaluate the cross section for pair production in the gravitational field of a cosmic string, and to discuss some of its potential consequences.

There is no Newtonian gravitational field around a static, straight cosmic string. The metric is locally flat. One might naively suspect impossible to have pair production by a single photon. This, however, is not the case, as was shown in a previous paper ${ }^{[2]}$ in the context of a simplified model based on a scalar field theory. The metric around a static straight string that lies along the z-axis reads, in cylindrical coordinates: ${ }^{[3,4]}$

$$
d s^{2}=d t^{2}-d \rho^{2}-\rho^{2} d \theta^{2}-d z^{2} .
$$

The metric is the same as in Minkowski space, but here the periodicity of the angular coordinate is within the range

$$
0 \leq \theta \leq \frac{2 \pi}{\nu}, \quad \text { with } \quad \nu=(1-4 G \mu)^{-1} .
$$

$\mu$ is the mass per unit length of string, and G is Newton's constant. The dimensionless quantity $\mathrm{G} \mu$ measures the strength of the gravitational effects of the string. The space-like sections around the string have the topology of a cone with the vertex at the core and with deficit angle $8 \pi \mathrm{G} \mu$. The conical structure of the cosmic string space-time is the source of momentum non-conservation in the plane perpendicular to the string, which allows for pair production by a single photon. This absence of global momentum conservation was already stressed for gravity in $2+1$ dimensions, ${ }^{[5]}$ where massive particles originate a conical structure of space-time, ${ }^{[6]}$ the same as strings do in $3+1$ dimensions.

The gravitational mechanism that permits pair production by a single photon around a cosmic string bears some resemblance with the Aharonov-Bohm effect, ${ }^{[7]}$ at least in its topological features. Recently the Aharonov-Bohm interaction of fermions with the pure gauge potentials around cosmic strings has been shown to lead to significantly large

scattering cross sections in models where the strings carry non-integer fluxes. ${ }^{[8,9,10,11]}$ The 
gravitational effect, though, is of a different nature than the Aharonov-Bohm effect, and is independent on the details of the field theoretical model for the cosmic string.

Classically, the gravitational effects of a straight cosmic string become manifest when two particles move along opposite sides of the string. Initially parallel trajectories converge as they move past the string, which then acts as a gravitational lens. ${ }^{[12,3]}$ The deflection angle is independent on the impact parameter. This peculiar features lead to the picture of a classical analogue of the Aharonov-Bohm effect. ${ }^{13]}$ Other classical effects may affect a single particle: the conical structure of the space-time induces a self-force, both gravitational as well as electrostatic, on a test particle around a string. ${ }^{[14,15]}$ Also, a freely moving charged particle radiates as it goes by a string, ${ }^{[16]}$ in analogy to the radiation by a charged particle when it suffers Aharonov-Bohm scattering. ${ }^{[17]}$ It must be noted that the conical singularity of the cosmic string metric (1), (2) is smoothed out in more realistic string models. In this sense the effects under consideration are not of topological origin but are caused by matter fields concentrated in cosmic strings.

This classical gravitational effects around a cosmic string give us a hint about the mechanism underlying pair production by a single photon. An heuristic, semiclassical picture follows. Virtual positron-electron pairs are continuously created from the vacuum, and quickly annihilate. A photon is not able to make them real in empty Minkowski space, even if it has sufficient energy, since otherwise momentum conservation would be violated. But in the presence of the string, the requisite momentum to make them real is provided by the string if each member of the pair moves along opposite sides. This picture may prove helpful to qualitatively understand some of the quantitative results we shall derive. Further properties of the pair production process, as well as extensions to others such as bremsstrahlung, were considered in Ref. 18 in the context of a scalar field theory. These problems in the framework of QED will be treated in a separate paper. ${ }^{[19]}$

This paper is organized as follows. In sections 2 and 3 we set up the stage by discussing the quantization of free electron and photon fields respectively in a cosmic string space-time. In section 4 we write up the QED interaction Lagrangian in the cosmic string gravitational background, and derive a closed expression for the matrix elements and cross section for pair production by a single high energy photon. We present analytic approximations valid at different energy regimes. In section 5 we round up the conclusions. 


\section{Dirac fields in a cosmic string space-time}

In this section we find the appropriate field operator for electrons and positrons in the conical space around a straight comic string. Similar studies of the Dirac equation were performed in $2+1$ dimensions, with two-component spinors, both in a conical space ${ }^{[20]}$ as well as in a vortex background, relevant for the Aharonov-Bohm effect. ${ }^{[8,9,10,11,21]}$ We shall work instead with usual four-component spinors in the $3+1$ dimensional space-time around a cosmic string, closer to the treatment of the Aharonov-Bohm effect performed in Ref. 22 .

Dirac equation in a curved space-time reads: ${ }^{[23]}$

$$
\left(i \gamma^{\mu}(x) \widetilde{\nabla}_{\mu}-M\right) \psi=0
$$

with $\gamma^{\mu}(x)=V_{(a)}^{\mu} \gamma^{a}$, where $V_{(a)}^{\mu}$ is a tetrad, and $\gamma^{a}$ are ordinary Dirac matrices, that satisfy $\gamma^{a} \gamma^{b}+\gamma^{b} \gamma^{a}=2 \eta^{a b}$. We work with the representation

$$
\gamma^{0}=\left(\begin{array}{cc}
1 & 0 \\
0 & -1
\end{array}\right), \gamma^{i}=\left(\begin{array}{cc}
0 & \sigma^{i} \\
-\sigma^{i} & 0
\end{array}\right)
$$

In eq.(3) the covariant derivative involves the spin connection $\Gamma_{\mu}$

$$
\widetilde{\nabla}_{\mu}=\partial_{\mu}+\Gamma_{\mu}, \quad \Gamma_{\mu}=\frac{1}{2} \Sigma^{a b} V_{(a)}^{\nu}\left(\nabla_{\mu} V_{(b) \nu}\right), \quad \Sigma^{a b}=\frac{1}{4}\left[\gamma^{a}, \gamma^{b}\right]
$$

For the cosmic string metric (1) we can take the tetrad as

$$
V_{(a)}^{\mu}=\left(\begin{array}{cccc}
1 & 0 & 0 & 0 \\
0 & c & s & 0 \\
0 & -s / \rho & c / \rho & 0 \\
0 & 0 & 0 & 1
\end{array}\right)
$$

where $c \equiv \cos (\nu \theta), s \equiv \sin (\nu \theta)$. Then the Dirac matrices $\gamma^{\mu}(x)$ in cylindrical coordinates read

$$
\gamma^{0}(x)=\gamma^{0}, \gamma^{3}(x)=\gamma^{3}, \gamma^{\rho}(x)=c \gamma^{1}+s \gamma^{2}, \gamma^{\theta}(x)=\frac{1}{\rho}\left(-s \gamma^{1}+c \gamma^{2}\right)
$$

and the spin connection is

$$
\Gamma_{\mu}=\delta_{\mu}^{\theta} \frac{\nu-1}{2} \gamma^{2} \gamma^{1}=\delta_{\mu}^{\theta} \frac{\nu-1}{2} i \Sigma_{3}
$$

Finally, the Dirac equation in the cosmic string metric reads

$$
\left(i\left[\gamma^{0} \partial_{t}+\gamma^{3} \partial_{z}+\gamma^{\rho}\left(\partial_{\rho}-\frac{\nu-1}{2 \rho}\right)+\gamma^{\theta} \partial_{\theta}\right]-M\right) \psi=0
$$


It is useful to write it also in the Hamiltonian form:

$$
i \partial_{t} \psi=H \psi, \quad \text { with } \quad H=\alpha^{i} p_{i}+\beta M
$$

where

$$
\alpha^{i}=\left(\begin{array}{cc}
0 & \sigma^{i} \\
\sigma^{i} & 0
\end{array}\right), \quad \beta=\gamma_{0}
$$

and

$$
p_{\rho}=-i \partial_{\rho}, \quad p_{\theta}=L_{3} \equiv-i \partial_{\theta}+\frac{\nu-1}{2} \Sigma_{3}, \quad p_{3}=-i \partial_{z}
$$

Here $\alpha^{\rho}$ and $\alpha^{\theta}$ are defined from $\alpha^{1}$ and $\alpha^{2}$ as for the Dirac matrices in (7). We can now write a complete set of commuting operators, and use their eigenvalues to label the quantum states of electrons and positrons:

$$
\begin{aligned}
& \hat{H} \psi=E \psi, \\
& \hat{p_{3} \psi}=p_{3} \psi, \\
& \hat{J}_{3} \psi=j_{3} \psi, \quad \hat{J}_{3}=L_{3}+\frac{1}{2} \Sigma_{3}, \quad j_{3}=\nu j, \\
& \hat{S}_{t} \psi=s \psi, \quad \hat{S}_{t}=\frac{1}{\sqrt{E^{2}-M^{2}}} \Sigma_{i} p_{i} .
\end{aligned}
$$

The solution to these equations representing an electron state with positive energy is

$$
\psi_{e}(j, x)=\frac{\sqrt{\nu}}{2 \pi} N_{e} \exp \left(-i E t+i p_{3} z\right)\left(\begin{array}{l}
u \\
v
\end{array}\right),
$$

with the two-component spinors $u, v$ given by

$$
\begin{aligned}
& u=\frac{1}{\sqrt{E-M}} \cdot\left(\begin{array}{c}
J_{\beta_{1}}\left(p_{\perp} \rho\right) \exp (i \nu l \theta) \\
\frac{i \epsilon_{l} s p_{\perp}}{p+s p_{3}} J_{\beta_{2}}\left(p_{\perp} \rho\right) \exp (i \nu(l+1) \theta)
\end{array}\right) \\
& v=\frac{1}{\sqrt{E+M}} \cdot\left(\begin{array}{c}
s J_{\beta_{1}}\left(p_{\perp} \rho\right) \exp (i \nu l \theta) \\
\frac{i \epsilon_{l} p_{\perp}}{p+s p_{3}} J_{\beta_{2}}\left(p_{\perp} \rho\right) \exp (i \nu(l+1) \theta)
\end{array}\right)
\end{aligned}
$$

and

$$
\begin{gathered}
N_{e}=\frac{\sqrt{p\left(p+s p_{3}\right)}}{2 \sqrt{E}}, \\
\beta_{1,2}=|\nu j \mp 1 / 2|, \quad j=l+1 / 2, \\
p_{\perp}=\sqrt{p^{2}-p_{3}^{2}}=\sqrt{E^{2}-M^{2}-p_{3}^{2}}, \\
\epsilon_{l}=\operatorname{sign}(l)= \pm 1, \quad\left(\begin{array}{c}
l \geq 0, \\
l<0
\end{array}\right)
\end{gathered}
$$


and $s= \pm 1$ is the value of $S_{t} . J_{\beta}$ are Bessel functions. Positron states $\psi_{p}$ with quantum numbers (11) can be obtained from electron states of negative energy through charge conjugation with the matrix $C=\alpha^{2}$ and by changing $p_{3} \rightarrow-p_{3}, j \rightarrow-j(l \rightarrow-l-1)$. So we find:

$$
\psi_{p}^{c}(j, x)=C \bar{\psi}_{p, t r a n s p}=\frac{\sqrt{\nu}}{2 \pi} N_{p}^{*} \exp \left(i E t-i p_{3} z\right)\left(\begin{array}{c}
y \\
w
\end{array}\right)
$$

where

$$
\begin{array}{r}
y=\frac{1}{\sqrt{E+M}} \cdot\left(\begin{array}{c}
J_{\beta_{2}}\left(p_{\perp} \rho\right) \exp (-i \nu(l+1) \theta) \\
\frac{-i \epsilon_{l} s p_{\perp}}{p-s p_{3}} J_{\beta_{1}}\left(p_{\perp} \rho\right) \exp (-i \nu l \theta)
\end{array}\right) \\
w=\frac{1}{\sqrt{E-M}} \cdot\left(\begin{array}{c}
-s J_{\beta_{2}}\left(p_{\perp} \rho\right) \exp (-i \nu(l+1) \theta) \\
\frac{i \epsilon_{l} p_{\perp}}{p-s p_{3}} J_{\beta_{1}}\left(p_{\perp} \rho\right) \exp (-i \nu l \theta)
\end{array}\right)
\end{array}
$$

with

$$
N_{p}=\frac{\sqrt{p\left(p-s p_{3}\right)}}{2 \sqrt{E}} .
$$

The electron-positron field operator is

$$
\psi(x, t)=\int d \mu_{j}\left[\psi_{e}(j, x) a_{j}+\psi_{p}^{c}(j, x) b_{j}^{\dagger}\right]
$$

where $a_{j}$ and $b_{j}$ are the annihilation operators for electrons and positrons with given quantum numbers. The normalization condition is

$$
\int d x \psi_{e, p}^{\dagger}(j, x) \psi_{e, p}\left(j^{\prime}, x\right)=\delta_{j, j^{\prime}}=\delta_{s, s^{\prime}} \delta_{l, l^{\prime}} \delta\left(p_{3}-p_{3}^{\prime}\right) \frac{\delta\left(p_{\perp}-p_{\perp}^{\prime}\right)}{\sqrt{p_{\perp} p_{\perp}^{\prime}}}
$$

We denote collectively by $j$ or $j^{\prime}$ the quantum numbers of a given state, and integration is also collectively denoted as

$$
\int d \mu_{j}=\sum_{l=-\infty}^{\infty} \int_{-\infty}^{\infty} d p_{3} \int_{0}^{\infty} p_{\perp} d p_{\perp}
$$

Before closing this section, it is worth pointing out that although the solution shares many similarities with that for fermions around a magnetic flux tube, there are also significant differences. An important distinction is that the parameter $\nu$, which deviates from unity in the conical space, appears multiplicatively in the order of the Bessel functions, while in the Aharonov-Bohm case the flux modifies additively the order of the Bessel functions. In connection with this, problems related with the non-self adjointness of the Dirac operator in the Aharonov-Bohm case ${ }^{[10,22,24]}$ do not seem to affect the present discussion. 


\section{Maxwell fields in a cosmic string space-time}

Maxwell equations in the cosmic string space-time with metric (1), in the Lorentz gauge, $\nabla_{\mu} A^{\mu}=0$, take the form

$$
\begin{aligned}
\square A_{\rho}-\frac{2}{\rho^{3}} \partial_{\theta} A_{\theta}-\frac{1}{\rho^{2}} A_{\rho} & =0 \\
\square A_{\theta}-\frac{2}{\rho} \partial_{\rho} A_{\theta}+\frac{2}{\rho} \partial_{\theta} A_{\rho} & =0 \\
\square A_{z}=\square A_{t} & =0,
\end{aligned}
$$

where

$$
\square=\Delta-\partial_{t t}^{2}, \quad \Delta=\frac{1}{\rho} \partial_{\rho}\left(\rho \partial_{\rho}\right)+\frac{1}{\rho^{2}} \partial_{\theta \theta}^{2}+\partial_{z z}^{2} .
$$

In order to decouple these equations, so as to identify the linear independent components to be quantized, we introduce spin-weighted components, defining ${ }^{[25]}$

$$
A_{\xi}=\frac{1}{\sqrt{2}}\left(A_{\rho}+\frac{i \xi}{\rho} A_{\theta}\right) \quad \text { if } \quad \xi= \pm 1 \quad ; \quad A_{\xi}=A_{z}, A_{t} \quad \text { if } \quad \xi=0
$$

The equations for the $A_{\xi}$ components decouple, and can be collectively written as

$$
\square_{\xi} A_{\xi}=0, \text { with } \square_{\xi}=\Delta_{\xi}-\partial_{t t}^{2},
$$

where

$$
\Delta_{\xi}=\frac{1}{\rho} \partial_{\rho}\left(\rho \partial_{\rho}\right)-\frac{1}{\rho^{2}} L_{3}^{2}+\partial_{z z}^{2}, \quad L_{3}=-i \partial_{\theta}+\xi
$$

The normal modes for the independent components are

$$
f_{\xi}(j, x)=\frac{\sqrt{\nu}}{2 \pi} \exp \left(i \nu m \theta+i k_{3} z\right) J_{|\nu m+\xi|}\left(k_{\perp} \rho\right) \frac{1}{\sqrt{2 \omega}} \exp (-i \omega t)
$$

where $j$ denotes collectively the values of $\left(k_{3}, k_{\perp}, m\right) . k_{3}$ is the eigenvalue of $\hat{k}_{z}=-i \partial_{z}$, and $l_{3}=\nu m+\xi$ is the eigenvalue of $L_{3}$. The modes are normalized according to

$$
\int d x f_{\xi}^{*}(j, x)\left(i \stackrel{\stackrel{\leftrightarrow}{\partial}_{t}}{)} f_{\xi}\left(j^{\prime}, x\right)=\delta_{j, j^{\prime}}\right.
$$

The total solution to the Maxwell equations is

$$
A_{\xi}(t, x)=\int d \mu_{j}\left(f_{\xi}(j, x) c_{\xi}+f_{-\xi}^{*}(j, x) c_{-\xi}^{\dagger}\right),
$$


or, back to cylindrical cordinates coordinates,

$$
\begin{aligned}
A_{\rho}(t, x) & =\int d \mu_{j} \frac{1}{\sqrt{2}}\left[\left(c_{+} f_{+}+c_{-} f_{-}\right)+\left(c_{+}^{*} f_{+}^{*}+c_{-}^{*} f_{-}^{*}\right)\right], \\
A_{\theta}(t, x) & =\int d \mu_{j} \frac{-i \rho}{\sqrt{2}}\left[\left(c_{+} f_{+}-c_{-} f_{-}\right)-\left(c_{+}^{*} f_{+}^{*}-c_{-}^{*} f_{-}^{*}\right)\right], \\
A_{z, t}(t, x) & =\int d \mu_{j}\left[c_{3,0} f_{0}+c_{3,0}^{*} f_{0}^{*}\right] .
\end{aligned}
$$

The solution with a given value of $\xi$ not only has a definite quantum number $l_{3}=\nu m+\xi$, but is also an eigenfunction of the spin z-component operator

$$
S_{3}=\left(\begin{array}{ccc}
0 & -i / \rho & 0 \\
i \rho & 0 & 0 \\
0 & 0 & 0
\end{array}\right)
$$

with quantum number $s_{3}=-\xi$. So, the photon wave functions

$$
A_{i}^{+}=\frac{1}{\sqrt{2}}\left(\begin{array}{c}
f_{+} \\
-i \rho f_{+} \\
0,
\end{array}\right), A_{i}^{-}=\frac{1}{\sqrt{2}}\left(\begin{array}{c}
f_{-} \\
i \rho f_{-} \\
0,
\end{array}\right), A_{i}^{0}=\left(\begin{array}{c}
0 \\
0 \\
f_{0}
\end{array}\right)
$$

have the following quantum numbers

$$
j_{3}=\nu m, \quad l_{3}=(\nu m \pm 1, \nu m), \quad s_{3}=(\mp 1,0) .
$$

The coefficients $c_{\xi}(j)$ can be promoted to operators with the commutation relations

$$
\left[c_{\xi}(j), c_{\xi}^{\dagger}\left(j^{\prime}\right)\right]=\delta_{j, j^{\prime}}
$$

These operators are annihilation and creation operators for a photon with quantum numbers $k_{\perp}, k_{3}, j_{3}(m), l_{3}(m), s_{3}$.

As a final remark, let us find out operators $c_{\sigma, \pi}$ which correspond to annihilation operators for physical, transverse photons, by explicitly working out the Lorentz gauge condition, which translates into:

$$
\frac{k_{\perp}}{\sqrt{2}}\left[\delta_{m 0}\left(c_{+}+c_{-}\right)+\epsilon_{m}\left(c_{+}-c_{-}\right)\right]+i\left(k_{3} c_{3}-\omega c_{0}\right)=0,
$$

where, as previously defined, $\epsilon_{m}=\operatorname{sign}(m)$. With $\vec{k}$ perpendicular to the string direction this determines annihilation operators for transverse photons as $c_{\sigma}=\frac{1}{\sqrt{2}}\left(c_{+}+c_{-}\right)$and $c_{\pi}=c_{3}$. 


\section{Cross section for pair production by a high energy photon}

\subsection{Matrix elements and cross section}

In this section we evaluate the matrix elements and cross section for electron-positron pair production by a single high energy photon moving in the flat but conical space-time around a cosmic string. This was already done for a simplified model based on scalar fields. ${ }^{[2,26,18]}$ The extension to QED is not so straightforward, as we have seen already in the previous sections. Actually, a very relevant difference appears in the energy dependence of the cross section at high energies, which is larger than in the corresponding scalar analog, and this may have significant consequences.

The QED interaction Lagrangian is

$$
L_{\text {int }}=-e \sqrt{-g} \bar{\psi}(x) A_{\mu}(x) \gamma^{\mu}(x) \psi(x)
$$

and we can write, in the notation of the previous sections:

$$
A_{\mu}(x) \gamma^{\mu}(x)=\sqrt{2} A_{+} \exp (i \nu \theta) \gamma^{+}+\sqrt{2} A_{-} \exp (-i \nu \theta) \gamma^{-}+A_{z}(x) \gamma^{3},
$$

where

$$
\gamma^{ \pm}=\frac{1}{2}\left(\gamma^{1} \mp i \gamma^{2}\right)=\left(\begin{array}{cc}
0 & \sigma_{ \pm} \\
-\sigma_{ \pm} & 0
\end{array}\right), \sigma_{+}=\left(\begin{array}{cc}
0 & 0 \\
1 & 0
\end{array}\right), \sigma_{-}=\left(\begin{array}{cc}
0 & 1 \\
0 & 0
\end{array}\right) .
$$

The matrix element for pair production of an electron with quantum numbers $j_{p}=$ $\left(p_{\perp}, p_{3}, l, s\right)$ and a positron with quantum numbers $j_{q}=\left(q_{\perp}, q_{3}, n, r\right)$ by a single photon with quantum numbers $j_{k}=\left(k_{\perp}, k_{3}, m, \lambda\right)$, can be written for physical states with $\lambda=\sigma, \pi$ in terms of matrix elements with $\xi= \pm 1,3$,

$$
M_{\xi}=<j_{p}, j_{q}\left|S^{(1)}\right| j_{k}>=-e \sqrt{2} \int d^{4} x \bar{\psi}_{e}\left(j_{p}, x\right) \gamma^{\xi} \psi_{p}^{c}\left(j_{q}, x\right) f_{\xi}\left(j_{k}, x\right) \exp (i \xi \nu \theta)
$$

that after a simple integration on $t, z$ and $\theta$ become

$$
M_{\xi}=-e \sqrt{\nu} \frac{\sqrt{p q}}{4 \sqrt{\omega E_{p} E_{q}}} \delta\left(\omega-E_{p}-E_{q}\right) \delta\left(k_{3}-p_{3}-q_{3}\right) \delta_{m, l+n+1} m_{\xi}
$$

where

$$
\begin{aligned}
& m_{+}=-i \epsilon_{l} \sqrt{\left(p-s p_{3}\right)\left(q-r q_{3}\right)} R J\left(\alpha_{+}, \beta_{+}\right) \\
& m_{-}=-i \epsilon_{n} s r \sqrt{\left(p+s p_{3}\right)\left(q+r q_{3}\right)} R J\left(\alpha_{-}, \beta_{-}\right), \\
& m_{3}=\frac{1}{\sqrt{2}}\left[r \sqrt{\left(p-s p_{3}\right)\left(q+r q_{3}\right)} J\left(\alpha_{+}, \beta_{-}\right)+s \sqrt{\left(p+s p_{3}\right)\left(q-r q_{3}\right)} J\left(\alpha_{-}, \beta_{+}\right)\right] R
\end{aligned}
$$


with

$$
R=\frac{1}{\sqrt{E_{p}+M} \sqrt{E_{q}+M}}-\frac{s r}{\sqrt{E_{p}-M} \sqrt{E_{q}-M}} .
$$

Here we denote

$$
J(\alpha, \beta)=\int_{0}^{\infty} d \rho \rho J_{|\alpha|}\left(p_{\perp} \rho\right) J_{|\beta|}\left(q_{\perp} \rho\right) J_{|\alpha+\beta|}\left(k_{\perp} \rho\right)
$$

with

$$
\alpha_{ \pm}=\nu\left(l+\frac{1}{2}\right) \pm \frac{1}{2}, \beta_{ \pm}=\nu\left(n+\frac{1}{2}\right) \pm \frac{1}{2} .
$$

For $k_{\perp}>p_{\perp}+q_{\perp}$ we obtain ${ }^{[27]}$

$$
J(\alpha, \beta)=\Theta(-\alpha \beta) \frac{2 \sin [\pi \min (|\alpha|,|\beta|)]}{\pi k_{\perp}^{2} \cos (A+B) \cos (A-B)}\left(\frac{\sin A}{\cos B}\right)^{|\alpha|}\left(\frac{\sin B}{\cos A}\right)^{|\beta|}
$$

where

$$
p_{\perp}=k_{\perp} \sin A \cos B, q_{\perp}=k_{\perp} \sin B \cos A
$$

We notice in (32) that energy as well as linear momentum along the string direction are of course conserved. The condition $m=l+n+1$ is the conservation law for the total angular momentum projection along the string direction

$$
j_{3}=\nu m=\nu\left(j+j^{\prime}\right), \quad j=l+\frac{1}{2}, \quad j^{\prime}=n+\frac{1}{2} .
$$

Notice in (34) the step function $\Theta(-\alpha \beta)=\Theta\left(-j j^{\prime}\right)$ in the matrix element. The members of the pair produced by a high energy photon must have opposite signs for their total angular momentum projections. This is also the case for scalar particles. ${ }^{[2]}$ An heuristic explanation for this fact arises in the framework of the semiclassical picture presented in section 1. Opposite signs for $j$ and $j^{\prime}$ represent, in a classical description, motion along opposite sides of the string, which is necessary to extract linear momentum away from it, and make real pairs out from virtual vacuum fluctuations. This, in some sense, implies a localization of the pair production mechanism in the neighborhood of the string core, as also discussed with a different approach in Ref.18

From the matrix element (31) we evaluate the differential probability per unit length of string and per unit time for the pair production process:

$$
W_{\lambda}=\frac{\nu e^{2} p q}{64 \pi^{2} \omega E_{p} E_{q}} \delta\left(\omega-E_{p}-E_{q}\right) \delta\left(k_{3}-p_{3}-q_{3}\right) \delta_{m, l+n+1}\left|m_{\lambda}\right|^{2},
$$


where

$$
\begin{gathered}
\left|m_{\sigma, \pi}\right|^{2}=\frac{8\left(E_{p} E_{q}-M^{2}-s r p q\right)}{\pi^{2} k_{\perp}^{4} p^{2} q^{2} \cos ^{2}(A+B) \cos ^{2}(A-B)} \cdot \\
\cdot\left[s \sqrt{\left(p-s p_{3}\right)\left(q \mp r q_{3}\right)} d\left(\alpha_{+}, \beta_{ \pm}\right) \mp r \sqrt{\left(p+s p_{3}\right)\left(q \pm r q_{3}\right)} d\left(\alpha_{-}, \beta_{\mp}\right)\right]^{2}, \\
d(\alpha, \beta)=\Theta(-\alpha \beta) \sin [\pi \min (|\alpha|,|\beta|)] a^{\frac{|\alpha|}{2}} b^{\frac{|\beta|}{2}} \\
a=\frac{\sin ^{2} A}{\cos ^{2} B}, b=\frac{\sin ^{2} B}{\cos ^{2} A} .
\end{gathered}
$$

Notice that the differential probability $W_{\xi}$ depends on the electron and positron polarizations mostly by the factor $\left(E_{p} E_{q}+M^{2}-s r p q\right.$ ) (and only in that way for pairs moving in the plane perpendicular to the string). Hence, pairs with opposite polarizations are produced with larger probability.

We are specially interested in the total cross section for pair production. To evaluate it, we need to integrate over final states. The sum over polarizations of the created particles is straightforward, and gives the factor

$$
\sum_{s, r}\left(p \mp s p_{3}\right)\left(q \mp r q_{3}\right)\left(E_{p} E_{q}+M^{2}-s r p q\right)=2 p q\left(k_{\perp}^{2}-p_{\perp}^{2}-q_{\perp}^{2}\right)
$$

The sum over angular momentum quantum numbers is more difficult to perform, but at the end we obtain

$$
\begin{aligned}
\sum_{l, n} \delta_{m, l+n+1} d^{2}\left(\alpha_{\xi}, \beta_{\xi^{\prime}}\right) & =\left\{\Theta(m)\left[a^{\frac{\xi}{2}} b^{-\frac{\xi^{\prime}}{2}} a^{\nu m}+a^{-\frac{\xi}{2}} b^{\frac{\xi^{\prime}}{2}} b^{\nu m}\right]\right. \\
& \left.+\Theta(-m)\left[a^{-\frac{\xi}{2}} b^{\frac{\xi^{\prime}}{2}} a^{-\nu m}+a^{\frac{\xi}{2}} b^{-\frac{\xi^{\prime}}{2}} b^{-\nu m}\right]\right\}(a b)^{\frac{\nu}{2}} \Sigma
\end{aligned}
$$

where

$$
\Sigma=\sum_{l=0}^{\infty} \cos ^{2}\left[\pi \nu\left(l-\frac{1}{2}\right)\right](a b)^{\nu l}=\cos ^{2} \frac{\pi \nu}{2} \frac{\left(1-a^{\nu} b^{\nu}\right)^{2}+8 a^{\nu} b^{\nu} \sin ^{2} \frac{\pi \nu}{2}}{\left(1-a^{\nu} b^{\nu}\right)\left[\left(1-a^{\nu} b^{\nu}\right)^{2}-4 a^{\nu} b^{\nu} \sin ^{2} \pi \nu\right]}
$$

Notice the factor $\cos ^{2} \frac{\pi \nu}{2}$ that vanishes, as it should, for $\nu=1$, when there is no deficit angle. It is different than the analogous factor $\sin ^{2}(\pi \nu)$ that appears in the case of pair production of scalar particles, ${ }^{[2]}$ and of the Aharonov-Bohm case (with the number of magnetic flux units playing the role of $\nu$ ). This distiction may be understood as the influence of the spin connection on the Dirac equation in curved metrics. 
Next we integrate on $d p_{3} d q_{3}$

$$
\begin{aligned}
\int_{-\infty}^{\infty} d p_{3} \int_{-\infty}^{\infty} d q_{3} & \frac{1}{E_{p} E_{q}} \delta\left(\omega-E_{p}-E_{q}\right) \delta\left(k_{3}-p_{3}-q_{3}\right)= \\
& \frac{4 \Theta\left[k_{\perp}^{4}-2 k_{\perp}^{2}\left(p_{\perp}^{2}+q_{\perp}^{2}\right)+\left(p_{\perp}^{2}-q_{\perp}^{2}\right)^{2}-4 k_{\perp}^{2} M^{2}\right]}{\sqrt{k_{\perp}^{4}-2 k_{\perp}^{2}\left(p_{\perp}^{2}+q_{\perp}^{2}\right)+\left(p_{\perp}^{2}-q_{\perp}^{2}\right)^{2}-4 k_{\perp}^{2} M^{2}}}
\end{aligned}
$$

and then we average on photon polarizations,

$$
\frac{1}{2} \sum_{l, n} \delta_{m, l+n+1} \sum_{\xi, \xi^{\prime}} d^{2}\left(\alpha_{\xi}, \beta_{\xi^{\prime}}\right)=\frac{a+b+1+a b}{\sqrt{a b}}(a b)^{\frac{\nu}{2}} \frac{a^{\nu|m|}+b^{\nu|m|}}{2} \Sigma .
$$

The final step would be the integration on $p_{\perp}$ and $q_{\perp}$, which can not be done analytically for arbitrary incoming photon energy. Thus we now introduce a convenient parametrization that facilitates analytic approximations in different energy regimes. We define the variables

$$
r=\frac{p_{\perp}^{2}+q_{\perp}^{2}}{k_{\perp}^{2}}, \quad t=\frac{p_{\perp}^{2}-q_{\perp}^{2}}{p_{\perp}^{2}+q_{\perp}^{2}}, \quad s=\sqrt{1-2 r+r^{2} t^{2}} .
$$

Integrated on $t$ and $s$, the expression for the total cross section takes the form

$$
\sigma_{m}=\frac{2 \nu e^{2}}{\pi^{4} \omega} \cos ^{2} \frac{\pi \nu}{2} \int_{0}^{1} d t \int_{\epsilon}^{1} d s B(s, t) f_{\nu}(a, b)
$$

where $\epsilon \equiv 2 M / k_{\perp}$ and

$$
\begin{gathered}
B(s, t)=\frac{1}{\sqrt{1-t^{2}}} \frac{1}{s \sqrt{s^{2}-\epsilon^{2}}} \frac{s^{2}+\sqrt{1-t^{2}\left(1-s^{2}\right)}}{\left[1+\sqrt{1-t^{2}\left(1-s^{2}\right)}\right]^{2}}\left[1-\frac{s^{2}\left(1-\sqrt{1-t^{2}\left(1-s^{2}\right)}\right)}{2 \sqrt{1-t^{2}\left(1-s^{2}\right)}}\right] \\
f_{\nu}(a, b)=\frac{a^{\nu|m|}+b^{\nu|m|}}{2}(a b)^{\frac{\nu}{2}} \frac{\left(1-a^{\nu} b^{\nu}\right)^{2}+8 a^{\nu} b^{\nu} \sin ^{2} \frac{\pi \nu}{2}}{\left(1-a^{\nu} b^{\nu}\right)\left[\left(1-a^{\nu} b^{\nu}\right)^{2}-4 a^{\nu} b^{\nu} \sin ^{2} \pi \nu\right]} .
\end{gathered}
$$

In terms of $r, t$ and $s$, the functions $a$ and $b$ read

$$
a=\frac{1+r t-s}{1+r t+s}, \quad b=\frac{1-r t-s}{1-r t+s} .
$$

Eq. (42) is our final closed expression for the cross section. 


\subsection{Approximations at different energy regimes}

We now analyze the expression (42) under different approximations. We will always consider the realistic case

$$
\nu \approx 1+\delta, \quad \text { with } \quad \delta=4 G \mu<<1
$$

Since $\delta$ is of order the mass per unit length in Planck units, it is reasonable to assume it is small (for instance it is of order $10^{-6}$ for GUT cosmic strings).

We first consider the low energy case, just above the threshold for pair production, with $\left(k_{\perp}-2 M\right) / 2 M<<1$. In this limit only the values of $s \approx 1$ contribute significantly to the integral in (42), and thus we may approximate $\epsilon \approx 1, \quad a \approx \frac{1}{2}(1-s)(1+t), \quad b \approx$ $\frac{1}{2}(1-s)(1-t)$, and then we find

$$
\sigma_{m} \approx \frac{e^{2} \delta^{2}}{8 \sqrt{2} \pi^{3 / 2} \omega}\left(\frac{k_{\perp}}{2 M}-1\right)^{\frac{3}{2}+|m|} \frac{\Gamma(|m|+1)}{\Gamma(|m|+5 / 2)} \quad \text { if } \quad \frac{k_{\perp}}{2 M}-1<<1 .
$$

At high energy, $k_{\perp}>>M$, (and $\epsilon<<1$ ) the main contribution in (42) arises from small values of $s$. In this case

$$
a \approx 1-s\left(1+\sqrt{\frac{1-t}{1+t}}\right), b \approx 1-s\left(1+\sqrt{\frac{1+t}{1-t}}\right)
$$

and one needs to be careful in finding the asymptotic behaviour. We get

$$
\sigma_{m} \approx \frac{e^{2} \delta^{2}}{2 \pi^{2} \omega} \int_{0}^{1} d t \int_{\epsilon}^{1} d s \frac{\left(1-t^{2}\right)^{\frac{3}{2}}\left(1+\sqrt{1-t^{2}}\right)^{-3}}{s^{2} \sqrt{s^{2}-\epsilon^{2}}\left[s^{2}\left(1+\sqrt{1-t^{2}}\right)^{2}+\pi^{2} \delta^{2}\left(1-t^{2}\right)\right]} .
$$

We still need to distinguish two different regimes at high energy. The term between square brackets in the denominator of (46) plays different roles whether $s$ can be smaller than $\delta$ or not. If the photon energy is not too high, $M<<k_{\perp}<<M / \delta$, we can neglect the term proportional to $\delta^{2}$, and then we find

$$
\sigma_{m} \approx \frac{e^{2} \delta^{2}}{1890 \pi^{2} \omega}\left(\frac{k_{\perp}}{M}\right)^{4} \quad \text { if } \quad M<<k_{\perp}<<\frac{M}{\delta}
$$

Finally, at ultrahigh energies, when $k_{\perp}>>\frac{M}{\delta}$, the term proportional to $\delta^{2}$ dominates and then we obtain

$$
\sigma_{m} \approx \frac{e^{2}}{60 \pi^{4} \omega}\left(\frac{k_{\perp}}{M}\right)^{2} \quad \text { if } \quad k_{\perp}>>\frac{M}{\delta}
$$


The cross section increases with increasing photon energy. More precisely, it increases with increasing $k_{\perp}$, since the dynamics in the direction parallel to the string is trivial. This rather peculiar energy dependence cannot continue unbounded, since at sufficiently large energies it would conflict with unitarity, which imposes a bound of order $\sigma_{m} \lesssim \omega^{-1}$ on the partial cross sections. The unitarity limit thus restricts the validity of eq. (47) up to energies $k_{\perp} \lesssim 10 M / \sqrt{e \delta}$, and that of eq. (48) up to $k_{\perp} \lesssim 100 M / e$. We think this is a consequence of the breakdown of the validity of perturbation theory at these high energies. Notice that for realistic values of $e$ and $\delta$, equation (48) for the ultrahigh energy behaviour of the cross section is always in confict with unitarity.

Notice that in both high energy regimes the cross section does not depend on the photon quantum number $m$ that determines its angular momentum projection along the string axis. This is actually only true up to some large value of $m$, of order $k_{\perp} / M$, after which the approximations we were making break down, and the cross section decreases with $m$. An heuristic, semiclassical explanation for this property may arise from the following observation. The classical analog of the cylindrical modes with given $m$ may be thought of as a flux of particles incident upon the string from all directions with radius of closest approach of order $\rho_{\min } \approx m / k_{\perp}$. [18] This will be shorter than the Compton wavelength $1 / M$ of the pair if $m<k_{\perp} / M$. In that case virtual members of a pair that move along opposite sides of the string, and thus are able to extract momentum from it, will get hit by the photon. All values of $m$ smaller than $k_{\perp} / M$ should then be equally efficient at producing pairs, while larger values should be less efficient.

\subsection{Cross section for "plane wave" photon states}

Equations (45), (47) and (48) give us the cross section for pair production in the gravitational field of a cosmic string by a single photon in an eigenstate of the angular momentum operator $L_{3}$ in eq.(18), with eigenvalue $l_{3}=\nu m \pm 1=j_{3} \pm 1$. These cylindrical normal modes we used proved to be rather convenient to carry out our calculations. However, it is more common and useful to express the cross section for incoming states of definite linear momentum, i.e. for plane waves. Of course, there are no exact plane wave solutions in this case, since the non-conservation of linear momentum in the plane perpendicular to the string is precisely the origin of the effect under consideration. It is possible, nevertheless, to define "almost" plane wave states, states that asymptotically represent the superposition of an incoming plane wave distorted by the scattered cylindrical 
wave. These are the states that should be used in place of standard plane waves. They were analysed in the context of classical and quantum scattering problems in conical $2+1$ dimensional space, both for scalar ${ }^{[28]}$ as well as for Dirac fields. ${ }^{[20]}$

It is not difficult to obtain the cross section for these "almost plane wave" states from the partial cross sections for states with given $j_{3}=\nu m$, that we already evaluated. We have shown how to do this in the case of scalar fields in Ref.26. Since its extension to the QED case is rather straightforward, at least if one is interested in the total cross section only, and not on its polarization dependence, let us simply outline the method developed for the scalar case. It is based on the fact that a normal mode with the asymptotic behaviour

$$
\psi(x) \approx \exp \left(i k_{\perp} \rho \cos \nu \theta\right)+f(\theta) \frac{\exp \left(i k_{\perp} \rho\right)}{\sqrt{\rho}},
$$

that represents an incoming plane wave distorted by the scattered outgoing cylindrical wave, may be expanded in partial waves of cylindrical type as

$$
\psi(x)=\sum_{m} c_{m} J_{|m| \nu}\left(k_{\perp} \rho\right) \exp (i m \nu \theta),
$$

where $c_{m}=\exp (i \pi m-i \pi|m| \nu / 2)$. Eq.(50) with $\nu=1$ is of course just the usual expansion of a plane wave in cylindrical modes. When $\nu$ is larger than one it incorporates the scattered wave, unavoidable as the "plane wave" propagates in the conical topology.

Using normal modes of this sort to characterize the quantum states, one can check that in the low energy regime the cross section for a plane wave incoming state is dominated by the s-partial wave, i.e. the mode with $m=0$ in (45). At high energies, on the contrary, all partial waves contribute with equal weight, so that $\sigma=\sum_{m} \sigma_{m}$, where we denote by $\sigma$ the total cross section for a plane wave state. This leads to a rather large enhancement of the cross section above the value of eqs.(47) and (48), since the contribution of each partial wave is basically independent on $m$ over a large range. Now we must work with a better degree of approximation than in the previous section, to find the behaviour for very large $m$ also. It is easier to perform the calculations doing the sum over $m$ before the integrals on $t$ and $s$ in eq.(42). One then finds that the integrand has one extra inverse power of $s$ than before the summation, and that leads to a cross section at high energies 
for plane wave incoming states that is a factor of order $k_{\perp} / M$ larger than eqs. (47) and (48). We get:

$$
\sigma=\sum_{m} \sigma_{m} \approx \frac{e^{2} \delta^{2}}{6720(2 \pi) \omega}\left(\frac{k_{\perp}}{M}\right)^{5} \quad \text { if } \quad M<<k_{\perp}<<\frac{M}{\delta}
$$

and, for the ultrahigh energy regime:

$$
\sigma=\sum_{m} \sigma_{m} \approx \frac{e^{2}}{60(2 \pi)^{3} \omega}\left(\frac{k_{\perp}}{M}\right)^{3} \quad \text { if } \quad k_{\perp}>>\frac{M}{\delta} .
$$

As mentioned in the previous section, these results are in conflict with the unitarity bound on the cross section if $k_{\perp} \gtrsim 10 M / \sqrt{e \delta}$ for eq. (51) and if $k_{\perp} \gtrsim 100 M / e$ for eq. (52), reflecting the breakdown of the perturbative expansion upon which this derivation is based.

\section{CONCLUSIONS}

We have shown that the lack of global linear momentum conservation in the plane perpendicular to a cosmic string, which is a consequence of its conical topology, permits electron-positron pairs to be produced by a single photon notwithstanding there is no local gravitational field. Expressions (45), (47), (48), (51) and (52) contain our quantitative results, with the cross section per unit length of string for this process at different energy regimes and for alternative incoming quantum states of the photon.

Previous results of a similar nature were already known for a simplified model based

on scalar fields. ${ }^{[2,26,18]}$ The extension to QED, though, proved not so straightforward, both in its technical details as well as in the energy dependence of the resulting cross sections. The scalar model was based on a Lagrangian $\lambda \varphi \psi^{2}$, with $\lambda$ a coupling constant with mass units, $\varphi$ a massless and $\psi$ a massive scalar field. The QED result corresponds to the scalar case with $\lambda$ replaced by $e k_{\perp}$. In the high energy regime this makes a significant difference.

The pair production process by a high energy photon in the space-time of a cosmic string can be regarded as a consequence of a kind of gravitational analog of the AharonovBohm effect. There is also a strictly speaking Aharonov-Bohm interaction of fermions with the gauge potential around a cosmic string, in models where the string carry noninteger fluxes. ${ }^{[8,10,11]}$ There are many similarities in the mathematical treatment of the Aharonov-Bohm interaction and of the gravitational effect discussed in this paper, but 
also substantial differences. Besides, the gravitational effect is quite insensitive to the details of the field theory behind the string.

It is very striking that the cross section for pair production at high energies, eq.(52), grows with the energy of the incoming photon. This behaviour should be cut-off at energies that probe the core of the string, $k_{\perp} \approx \sqrt{\mu}$, where the metric is not well approximated by that of eq.(1), which has a conical singularity at the origin. A real cosmic string has a smooth core, and its metric approaches a flat, regular metric at the origin. The metric is that of a snub-nosed cone. ${ }^{[29,30]}$ It was shown, for instance, that the $1 / \rho$ self-force that a charged particle experiences in the conical space-time around a string, ${ }^{[14,15]}$ is cut-off at a distance of order the core radius in the snub-nosed cone metric. ${ }^{[31]}$ However, the unitarity bound on the cross section is already violated at energies much lower than those which probe the string core, reflecting the breakdown at high energies of the perturbative approach used to derive our results for the cross section.

\section{Acknowledgments}

D.H. is grateful to the Lebedev Physical Institute and especially to V. Frolov and the other members of the Relativity group for their hospitality. V.S. thanks M. Castagnino and the University of Buenos Aires as well as J.Audretsch, A.Economou and the other members of the Relativity group at the University of Konstanz for hospitality, collaboration and many fruitful discussions.

This work was supported by the Deutsche Forschungsgemeinschaft, the European Community DG XII, CONICET and Fundación Antorchas.

\section{REFERENCES}

1. H. Bethe and W. Heitler, Proc. Roy. Soc. A 146, 83 (1934)

2. D.D. Harari and V.D. Skarzhinsky, Phys. Lett. B 240, 322 (1990)

3. A. Vilenkin, Phys. Rep. 121, 263 (1985)

4. J. Gott, Ap. J. 288, 422 (1985)

5. M. Henneaux, Phys. Rev. D 29, 2766 (1984); S. Deser, Class. Quantum Grav. 2, 489 (1985) 
6. S. Deser, R. Jackiw and G. 't Hooft, Ann. Phys. 152, 220 (1984)

7. Y. Aharonov and D. Bohm, Phys. Rev. 119, 485 (1959)

8. M.G. Alford and F. Wilczeck, Phys. Rev. Lett. 62, 1071 (1989)

9. M.G. Alford, J. March-Russell and F. Wilczeck, Nucl. Phys. B 328, 140 (1989)

10. Ph. de Sousa Gerbert, Phys. Rev. D 40, 1346 (1989)

11. W.B. Perkins, L. Perivolaropoulos, A.C. Davis, R.H. Brandenberger and A. Matheson, Nucl. Phys. B 353, 237 (1991)

12. A. Vilenkin, Phys. Rev. D 23, 852 (1981)

13. L.Ford and A. Vilenkin, J. Phys. A 14, 2353 (1981)

14. B. Linet, Phys. Rev. D 33, 1833 (1986)

15. G. Smith, in "Formation and evolution of cosmic strings", Proceedings of the Cambridge Workshop, ed. by G.W. Gibbons, S.W. Hawking and T. Vachaspati, Cambridge Univ. Press (1990).

16. V.P. Frolov, E.M. Serebryany and V.D. Skarzhinsky, in Proceedings of the 4th Moscow Seminar on Quantum Gravity, ed. by M.A. Markov, V.A. Berezin and V.P. Frolov, World Sci. (1988).

17. E.M. Serebryany and V.D. Skarzhinsky, "The electromagnetic radiation at the Aharonov-Bohm scattering" in Proceedings of the Lebedev Physical Institute 197 (1989) 181.

18. J. Audretsch and A. Economou, Phys. Rev. D 44, 980 (1991) and Phys. Rev. D 44, 3774 (1991); J.Audretsch, A. Economou and D. Tsoubelis, Phys. Rev. D 45, 1103 (1992) 
19. J. Audretsch, U. Jasper and V. Skarzhinsky, in preparation.

20. Ph. de Sousa Gerbert and R. Jackiw, Comm. Math.Phys. 124, 229 (1989)

21. C.R. Hagen, Phys. Rev. Lett. 64, 503 (1990) and Phys. Rev. D 41, 2015 (1990)

22. S.A. Voropaev, D.V. Galtsov and D.A. Spasov, Phys. Lett. B 267, 91 (1991); Europhys. Lett. 12, 609 (1990)

23. S. Weinberg, Gravitation and Cosmology, Wiley, N.Y. (1972)

24. B.S. Kay and U.M. Studer, Commun. Math. Phys. 139, 103 (1991)

25. A.N. Aliev and D.V. Gal'tsov, Annals of Physics 193, 142 (1989)

26. D.D. Harari and V.D. Skarzhinsky, in "Proceedings of the $V$ Seminar on Quantum Gravity", Moscow 1990, ed. by M.A. Markov, V.A. Berezin and V.P. Frolov, World Scientific (1991); and in "Proceedings of First International A. D. Sakharov Conference in Physics", Moscow (1991).

27. I.S. Gradshteyn and I.M. Ryzhik, Table of integrals, series and products, Academic Press (1980).

28. S. Deser and R. Jackiw, Comm. Math. Phys. 118, 495 (1988); G.'t Hooft, Comm. Math. Phys. 117, 685 (1988)

29. R. Gregory, Phys. Rev. Lett. 59, 740 (1987)

30. D. Garfinkle, Phys. Rev. D 32, 1323 (1985)

31. W.B. Perkins and A.C. Davis, Nucl. Phys. B 349, 207 (1991) 